\title{
Vers une modélisation opérationnelle pour l'aménagement des zones côtières : l'exemple du bassin de Marennes-Oléron (France)
}

\author{
Towards an operational modelling for coastal zones management : \\ the example of Marennes-Oléron bay (France)
}

par C. Bacher, S. Sochard, CREMA*

C. Freissinet, P. Sauvaget, SOGREAH

An operational modelling strategy has been implemented for Marennes-Olerron bay (France) within the OPCOM European project. The strategy is based on the following points : $i)$ definition of the objectives of an operational model based on inquiries among potential end-users and decision-makers, ii) implementation and validation of TELEMAC2D software to simulate the hydrodynamism and the transport of suspended and dissolved matter, iii) development of an interface between the model outputs and a Geographic Informations System (GIS) to enable the visualisation and the analysis of the model results. The spatial and temporal variability of basic parameters - current velocity and direction, water elevation, salinity, was therefore assessed through several simulations based on different scenarios of wind, tidal coefficient and river freshwater flows. The model outputs were imported in a GIS and statistical tools implemented in order to combine the information yielded by the model with other existing information and to help in coastal zone management.

\section{I — INTRODUCTION}

Le but principal du projet OPCOM (OPerational modelling for COastal zone Management - Mast 3 - site Web: http://www.hydromod.de/projects/opcom/opcom.html) consiste à améliorer les méthodes de prévision en milieu côtier en évaluant essentiellement la capacité d'outils existants à répondre à des besoins de type opérationnel dans quatre sites sur lesquels ces outils ont été développés et testés par les partenaires du projet. Il s'agit de l'estuaire de l'Elbe (Allemagne), du bassin de Marennes-Oléron (France), de l'estuaire du Tage (Portugal) et de l'Archipelago Sea (Finlande). Les objectifs, les modèles et leur application sont spécifiques à chacun des sites mais leur évaluation répond à une démarche commune basée sur des enquêtes auprès des utilisateurs potentiels et décideurs afin de définir les besoins en gestion de la zone côtière, les spécifications minimales en matière de quantité et qualité des données requises pour l'implantation des modèles (assimilation des données) et leur validation, et les capacités de prévision à moyen et à long termes dans une perspective de gestion de la zone côtière.

Le bassin de Marennes-Oléron a été choisi en France du fait des enjeux économiques et des connaissances scientifiques accumulées au cours des années sur les plans hydrologique, hydrodynamique, sédimentaire et biologique et que l'on trouve dans de nombreuses études, thèses, articles et documents techniques. Le bassin de Marennes-Oléron est une baie d'environ $40 \mathrm{~km}$ dans le sens nord/sud et $15 \mathrm{~km}$ dans le sens ouest/est, enserrée entre l'île d'Oléron à l'ouest, l'île de Ré au Nord et le continent à l'est et communiquant avec l'Océan Atlantique par des Pertuis (Antioche au nord, Maumusson au sud - Figure 1). C'est une baie de type macrotidal dont le marnage dépasse $5 \mathrm{~m}$. Il s'agit du premier site français de production conchylicole, avec un stock

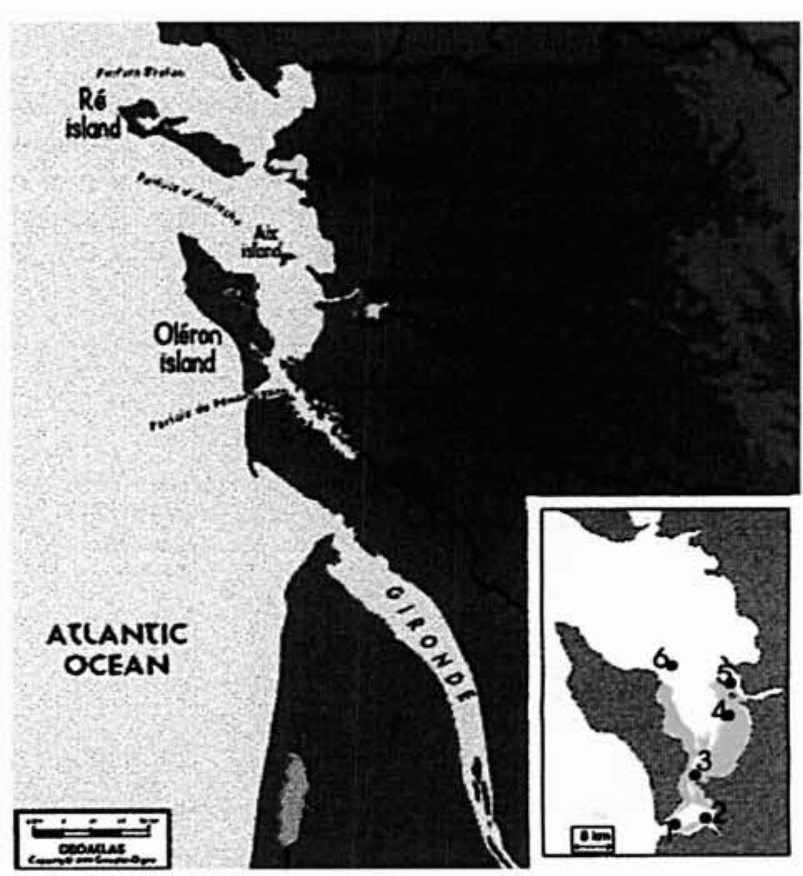

1. Carte des Pertuis Charentais, et sites du réseau d'observation hydrobiologique de I'IFREMER.

•BP 5, 17137 L'Houmeau, e-mail : cbacher@ ifremer.fr. 
d'huîtres japonaises et de moules en élevage d'environ 100000 tonnes, pour une production annuelle autour de 30000 tonnes et des emplois directs et indirects générés par cette activité qui se chiffrent en milliers. Cette filière traditionnelle soumise depuis longtemps à des réglementations nationales et plus récemment européennes est fragilisée d'une part du fait d'un marché de concurrence forte entre bassins de production nationaux et européens, et d'autre part, comme toute culture extensive, aux variations climatiques et environnementales qui influent sur la qualité et la quantité des coquillages produits. Ce bassin est également un site géographique d'importance touristique notable et, dans son voisinage, s'est développée une agriculture intensive ayant un impact sur le milieu par l'intermédiaire du réseau hydrologique du bassin versant de la Charente et de marais salés traditionnellement développés dans la frange littorale, activités économiques parmi d'autres (réserves ornithologiques, pêche, commerce maritime, aquaculture en marais par exemple) qui concourent à la nécessité de définir des politiques de gestion adéquates de la zone côtière. C'est ainsi que des études récentes ont permis d'évaluer certains impacts des zones de marais sur les eaux du bassin de Marennes-Oléron [1, 2] ou des apports de Charente sur la productivité côtière [3].

Dans le cadre du projet OPCOM, on se limite à la partie maritime de la zone côtière et, s'agissant d'un milieu à l'écologie complexe, on a cherché à définir un outil minimum pouvant répondre à des questions de prévision communes posées par différents interlocuteurs locaux. Ceux-ci sont impliqués dans les études d'impact d'aménagements littoraux, la protection de l'environnement ou le maintien des activités économiques du bassin. Ils sont notamment intéressés par la variabilité à long terme de certains paramètres environnementaux (température, salinité, turbidité) et des caractéristiques hydrodynamiques qui s'avèrent être les critères de référence pour la gestion du domaine maritime. Les questions dépassent cependant ce cadre minimum, et les préoccupations suivantes sont données à titre d'exemple de problèmes de gestion et d'aménagement :

- impact de l'hydrologie des marais salés sur les zones intertidales. Les marais salés sont alimentés par les variations du niveau marin et sont utilisés pour l'aquaculture intensive et extensive. Les lâchers d'eau liés à leur gestion hydraulique peuvent avoir un impact négatif (augmentation brutale de salinité, baisse de l'oxygène dissous) sur les élevages conchylicoles situés sur les zones découvrantes du littoral avoisinant (estran).

- développement de nouvelles techniques de conchyliculture. La recherche de secteurs aquacoles plus productifs conduit les professionnels de la conchyliculture à identifier les facteurs favorables/défavorables : bathymétrie, vitesse des courants, houle, salinité, qualité de l'eau.

- entretien des secteurs conchylicoles et des chenaux de navigation existants. Certains secteurs sont soumis à un envasement qui nécessite des opérations de dragage régulières, d'où les questions récurrentes de zones de relargage minimisant l'impact des dragages.

- impact des apports du bassin versant sur la qualité des eaux côtières, notamment dans la perspective d'une aquaculture durable. Certains apports des rivières sont nécessaires pour maintenir la productivité du bassin (apports en nitrates dans certaines limites), la conchyliculture elle-même nécessite, aux périodes de reproduction par exemple, des débits d'eau douce soutenus. Les contrôles sanitaires de la qualité des coquillages sont imposés par l'occurrence d'apports terrigènes à fort niveau bactérien. Les produits phytosanitaires utilisés à grande échelle par l'agriculture peuvent également avoir un impact négatif sur la conchyliculture. Des conflits d'usage sont ainsi générés par les besoins antagonistes en amont de la zone côtière et de l'aquaculture.

- problème de surcote et de crue. Dans cette région où le trait de côte ne cesse d'évoluer sous l'influence des phénomènes sédimentaires et des variations du niveau moyen des mers, certains secteurs à terre doivent être protégés des surcotes par des digues. Parallèlement, les débits de crue peuvent provoquer des inondations massives et conduisent à instrumenter l'embouchure de certains cours d'eau (Charente) pour évaluer en temps réel les débits accidentels.

La modélisation opérationnelle des paramètres hydrodynamiques, du transport de substances conservatives et, à plus long terme, de la dynamique sédimentaire contribue ainsi à la définition des zones littorales sensibles. Elle doit s'appuyer sur l'analyse des mécanismes physiques en jeu (conceptualisation et définition de cas types), l'implémentation des outils de simulation appropriés et leur utilisation pour des analyses de risque. Les paramètres-clés dépendent des apports de rivières, des paramètres météorologiques et climatiques, et de la géomorphologie.

Un modèle hydrodynamique a été mis en place, validé et testé par SOGREAH et une base de données géographiques couplée aux résultats obtenus à partir de scénarios de simulation. Des simulations sont présentées pour un certain nombre de scénarios afin de montrer la variabilité à court terme de la circulation des masses d'eau et l'importance d'un certain nombre de facteurs : vent, débit des estuaires, coefficient de marées, dont la variabilité est tout d'abord résumée et analysée d'après les données disponibles. L'intérêt de la construction d'une base de données incluant les paramètres simulés repose sur la possibilité d'interrogation (requêtes) des résultats pour établir des recoupements avec d'autres informations. Cela est rendu possible grâce à la réalisation d'une interface d'importation et d'analyse des simulations dans un SIG (Système d'Informations géographiques) dont les principes et certains résultats sont détaillés.

\section{II - VARIABILITÉ TEMPORELLE ET SPATIALE}

Un réseau de suivi de paramètres hydrobiologiques a été mis en place par l'IFREMER depuis 1977. Ce réseau est composé d'un certain nombre de stations échantillonnées régulièrement environ deux fois par mois en fonction de la marée. On distinguera deux fonctions principales à ce jeu de données : le suivi à long terme de paramètres-clés $[4,5]$, la fourniture d'informations aux études portant sur l'écosystème marin. On résumera ici certaines des variables mesurées : température, salinité, afin de montrer les échelles de variabilités spatiale et temporelle.

La température est relativement uniforme et les gradients spatiaux éventuels sont négligeables par rapport à la variabilité saisonnière. La température varie ainsi de $8^{\circ} \mathrm{C}$ à $20^{\circ} \mathrm{C}$ durant l'année avec des moyennes hivernale (resp. estivale) de $11^{\circ} \mathrm{C}$ (resp. $19^{\circ} \mathrm{C}$ ), mais la variabilité interannuelle peut atteindre $10^{\circ} \mathrm{C}$. La salinité est beaucoup plus variable dans l'espace du fait de l'influence de la Charente. La Figure 2 présente l'histogramme de toutes les valeurs observées sur l'ensemble des sites que l'on trouve généralement dans une 


\begin{tabular}{|c|c|c|c|c|c|}
\hline \multirow{2}{*}{ Rivière } & longueur $(\mathrm{km})$ & \multicolumn{3}{|c|}{ Débit } & $\begin{array}{c}\text { Débit annuel } \\
\left(\mathrm{m}^{3}\right)\end{array}$ \\
\cline { 2 - 6 } & & Moyenne $\left(\mathrm{m}^{3} / \mathrm{s}\right)$ & Crue $\left(\mathrm{m}^{3} / \mathrm{s}\right)$ & Etiage $\left(\mathrm{m}^{3} / \mathrm{s}\right)$ & $1,1 \times 10^{9}$ \\
\hline Charente & 45 & 36,1 & 600 & 13 & $3,2 \times 10^{7}$ \\
\hline Seudre & 20 & 1,01 & 8,9 & 0,037 & $2,9 \times 10^{10}$ \\
\hline Gironde & 70 & $\begin{array}{c}\text { Garonne }: 631 \\
\text { Dordogne }: 284\end{array}$ & $\begin{array}{c}\text { Garonne }: 5700 \\
\text { Dordogne }: 2600\end{array}$ & $\begin{array}{c}\text { Garonne }: 39 \\
\text { Dordogne }: 8,45\end{array}$ \\
\hline
\end{tabular}

Tableau 1. - Débits moyens, de crue et d'étiage sur trois rivières.

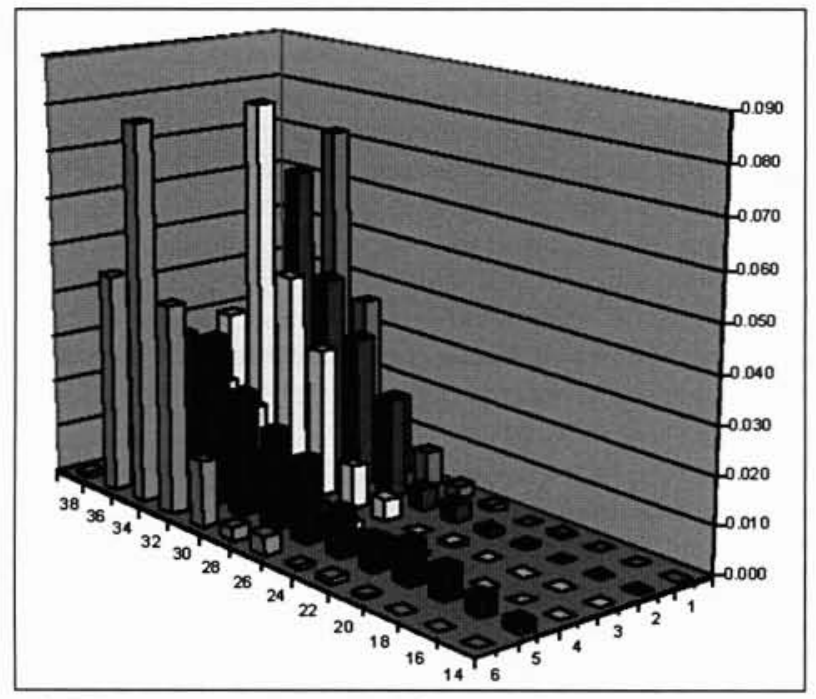

2. Histogramme des salinités observées sur l'ensemble des sites du réseau d'observation IFREMER.

gamme allant de 30 à $36 \%$, mais les sites 4 et 5 sont très sensibles aux apports d'eau douce et de fortes dessalures sont observées en hiver. Les sites 5 (embouchure de la Charente) et 1 (sud du bassin, dominé par l'influence des eaux océaniques) ont des profils très différents (figure 3 ). Saisonnalité et variabilité interannuelles sont plus prononcées au site 5 où la salinité peut descendre en dessous de $10 \%$. La variabilité temporelle est également importante à plus court terme, pendant le cycle mortes-eaux/vives eaux de 15 jours, du fait du lien classique entre marnage, courants de marée et intensités de mélange des eaux d'origines océanique et continentale. Les apports d'eau douce résultent de l'influence de trois estuaires: Seudre (partie sud du bassin), Charente (est), Gironde (au sud) dont les débits sont résumés dans le tableau 1 (source Banque Hydro). Le débit annuel de la Seudre repésente 3\% du débit annuel de la Charente [6]. Seule une fraction de la Gironde entre dans le bassin de Marennes-Oléron par le Pertuis de Maumusson principalement [7] et la Charente représente ainsi le principal apport d'eau douce, dont on retrouve l'influence saisonnière sur la variabilité spatiale et temporelle de la salinité.

A l'influence des débits des estuaires sur la circulation des masses d'eau et leur mélange, il faut sans doute ajouter l'effet du vent dont les directions et vitesses sont mesurées par la Météorologie Nationale en deux sites toutes les trois heures. La synthèse des données sur 10 années (1977-1986) montre les fréquences principales des différentes direction et vitesse (Figure 4).

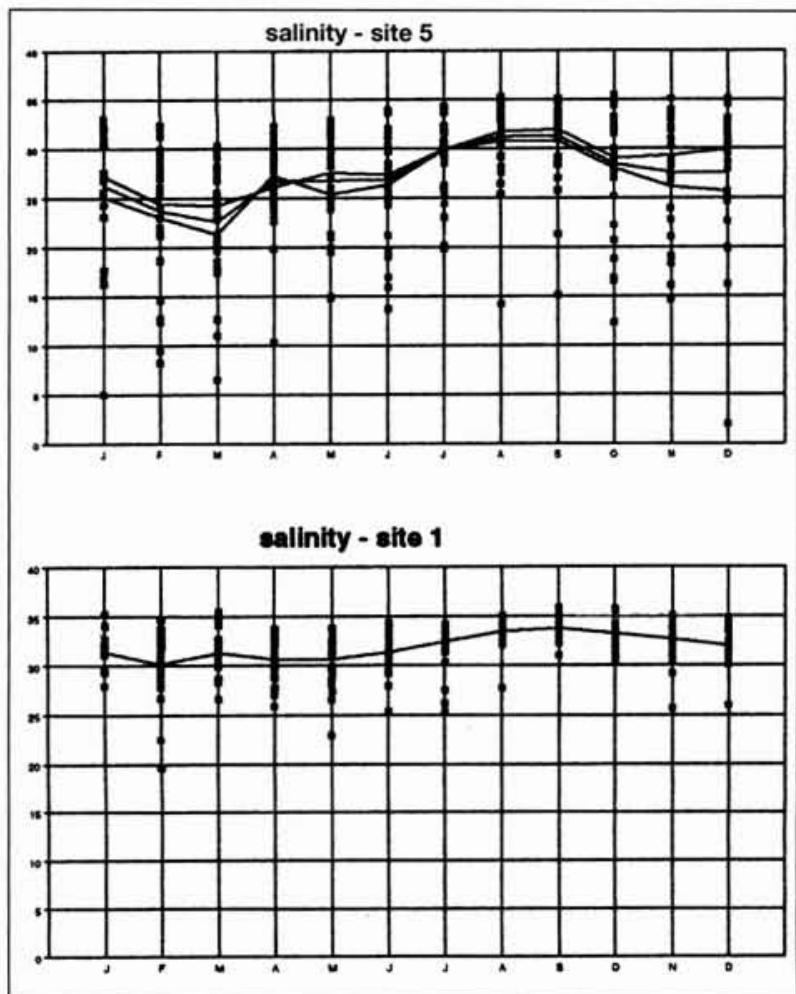

3. Variabilité temporelles des salinités en deux points du réseau d'observation IFREMER, dans l'embouchure de la Charente (point 5 ) et au sud du bassin (point 1).

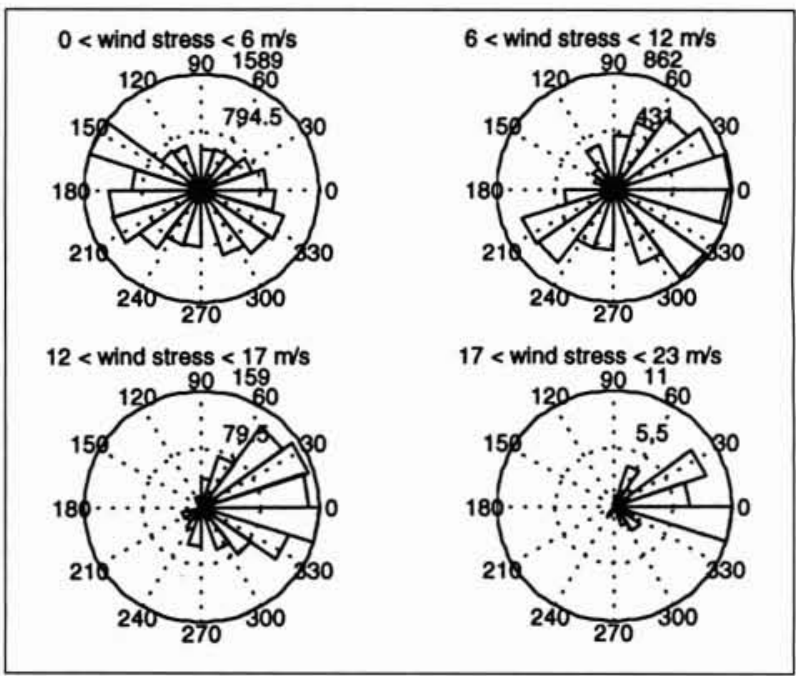

4. Statistiques des intensités et direction du vent établies sur 10 années de mesures. 


\section{III — MODÈLE HYDRODYNAMIQUE}

Le code de calcul TELEMAC-2D développé par le Laboratoire National d'Hydraulique et d'Environnement (EDFLNHE) a été utilisé pour réaliser les simulations. Basé sur un schéma numérique aux éléments finis, il résoud les équations de Navier-Stokes sur un maillage curviligne [8]. Un autre programme résoud les équations de transport de traceurs conservatifs (SUBIEF-2D). Un premier maillage recouvre l'ensemble du Golfe de Gascogne, de la pointe de Penmarch à la péninsule ibérique, et fournit les conditions limites d'un maillage plus fin qui s'étend du sud de la Gironde jusqu'au Sables d'Olonne en englobant la zone des Pertuis Charentais, soit un secteur de $120 \mathrm{~km}$ dans la direction Nord/Sud sur $80 \mathrm{~km}$ dans la direction Est/Ouest (Figure 1).

Pour le modèle à grande emprise, la bathymétrie utilisée provient des bases de données GEBCO 97 (General Bathymetric Chart of the Oceans) pour le plateau continental et du SHOM pour la zone côtière. Les conditions aux limites sont définies en élévation et phases de la marée à partir de la base de données LPAL 94 [9] développée par le LEGI (Laboratoire des Ecoulements Geophysiques et Industriels, Université de Grenoble). Le modèle a été calibré en comparant les harmoniques calculées sur les simulations et les harmoniques établies sur un réseau de stations [10] en corrigeant principalement la bathymétrie dans la frange 100-200 m, imprécise et à laquelle le modèle s'est trouvé très sensible.

Pour le modèle local, les bases de données utilisées pour la bathymétrie sont celles du SHOM, de la DDE-17 (Direction Départementale de l'Equipement de Charente-Maritime), et de l'IGN. Ces différentes données sont très hétérogènes : il existe quelques relevés bathymétriques partiels en Charente datant des années 1970 qui couvrent essentiellement les 20 premiers kilomètres de l'embouchure ; les relevés sont quasiment inexistants pour l'embouchure de la Seudre ; la partie Sud du bassin, entre l'île d'Aix et le Pertuis de Maumusson, est la mieux renseignée, des campagnes de relevés denses ayant été effectuées par la DDE-17 en 1995 et 1996 - ces relevés ne concernent cependant pas une partie importante des zones d'estran et une interpolation spatiale a dû être faite. Les débits des rivières Seudre, Charente et de la Gironde sont issus de la Banque Hydro nationale. La calibration du coefficient de frottement a permis d'obtenir des bonnes prédictions des niveaux d'eau en comparaison des enregistrements disponibles aux Sables d'Olonne, à La Rochelle, l'Ile d'Aix, la Pointe de Gatseau (SHOM) et en Charente (SOGREAH) [10]. La comparaison d'images LANDSAT2 du panache turbide de la Gironde [11] à plusieurs dates au panache de dessalure simulée par le modèle dans les conditions de débits de rivière et de coefficients de marée similaires a montré que les 2 panaches avaient la même extension géographique [10]. Il s'agit d'une validation qualitative du modèle de transport dans la mesure où l'on ne simule pas la dynamique sédimentaire (érosion/sédimentation) qui intervient dans le transport des matières en suspension.

L'étude de la variabilité spatiale et temporelle des caractéristiques physiques courant, salinité, température, est basée essentiellement sur l'analyse de leur sensibilité aux forçages principaux : débits, coefficient de marée, vent. Les scénarios suivants sont basés sur les variations à long terme des forçages et ont donné lieu à autant de simulations de l'évolution à court terme (1 mois) :

- Débits moyens et marée de vives-eaux/Débits moyens et marée de mortes-eaux,

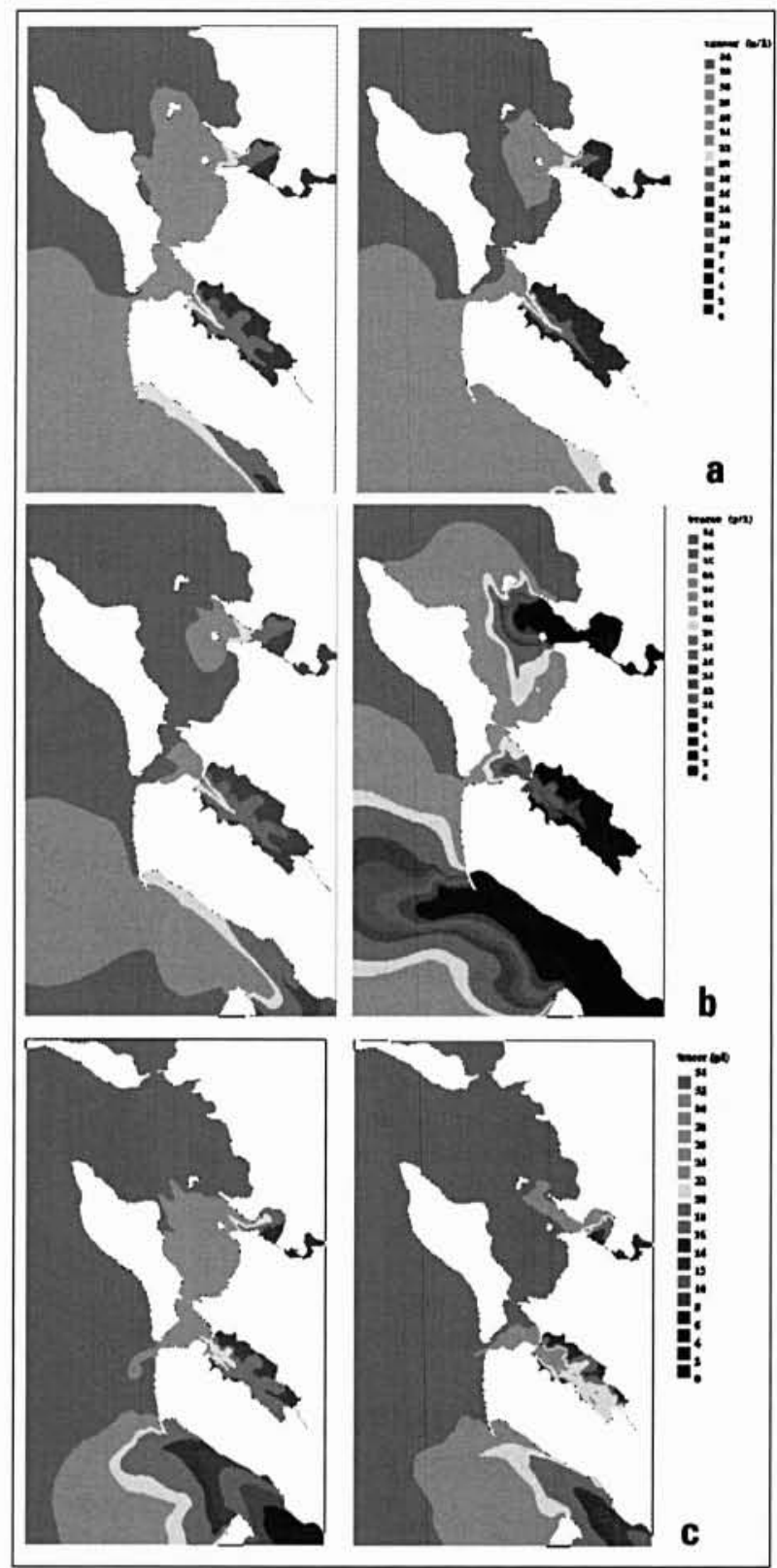

5. Simulation de la salinité pour différents scénarios. a) marées de vives-eaux et mortes eaux avec débits moyens de rivières ; b) marée de vives-eaux avec débits moyens et débits de crue ; c) vents d'ouest et de nord-ouest.

- Débits de crue et marée de vives-eaux/Débits de crue et marée de mortes-eaux,

- Débits moyens, vent d'ouest $(20 \mathrm{~m} / \mathrm{s})$ et marée de viveseaux,

- Débits moyens, vent de nord-ouest $(12 \mathrm{~m} / \mathrm{s}$ ) et marée de vives-eaux.

Les courants de marée et le marnage sont nettement plus importants en marée de vives-eaux qu'en mortes-eaux. Le coefficient de marée joue ainsi sur l'intensité du mélange entre les masses d'eau et notamment sur la dilution des panaches d'eau douce et le temps de résidence des masses d'eau dans le bassin (Figure 5). L'influence du vent est égale- 
ment très importante pour la position et l'étendue des panaches qui peuvent être considérablement modifiés par l'intensité et la direction du vent. Les gradients de salinité sont ainsi marqués non seulement dans l'embouchure de la Charente mais également dans les zones conchylicoles du nord du bassin, avec une influence plus marquée en général sur les estrans est que sur ceux au niveau de l'île d'Oléron. Cette cartographie instantanée des résultats ne doit pas masquer l'aspect dynamique de l'évolution des paramètres physicochimiques. Les maxima de courant, même s'ils dépendent du coefficient de marée, du régime hydrologique et des conditions météorologiques, sont fréquemment supérieurs à $1 \mathrm{~m} / \mathrm{s}$ à l'intérieur du bassin. Les trajectoires des masses d'eau ont une composante résiduelle orientée vers le sud, elles s'évacuent préférentiellement par le Pertuis de Maumusson et leur temps de résidence dans le secteur délimité par l'île d'Aix au nord et le Pertuis de Maumusson au sud est inférieur à 10 jours. L'excursion de marée, distance parcourue pendant un cycle de marée semi-diurne, atteint plusieurs kilomètres au nord du Chapus, qui est la partie centrale du bassin. La Charente est la rivière ayant le plus d'effet sur les gradients de salinité dans le bassin, alors que la Seudre, de débit plus faible et dont l'embouchure est située dans le sud du bassin où le courant de marée sont moins intenses qu'au centre, n'a qu'une influence locale. Le panache de la Gironde remonte le long de l'île d'Oléron et son influence peut se faire sentir, selon les conditions de vent et l'heure de la marée, au sud de Marennes-Oléron.

\section{IV — SYSTÈME D'INFORMA- TION GÉOGRAPHIQUE}

L'IFREMER a développé un Système d'Information Géographique basé sur le logiciel Arcview et ses extensions qui permet d'entrer dans une même base des informations géoréférencées : bathymétrie, cadastre conchylicole, fonds de carte IGN... Les coordonnées géographiques sont toutes exprimées de façon standardisée (projection LAMBERT). Le trait de côte est ainsi de type ligne, dont une propriété (attribut) est la longueur. La bathymétrie est entrée sous forme d'une grille régulière de points à partir desquels on peut calculer des isobathes ou estimer une valeur ponctuelle par interpolation. Le cadastre conchylicole est constitué de polygones disjoints ayant chacun une superficie connue. L'analyse de ces données hétérogènes consiste à visualiser, calculer les statistiques, combiner les différents thèmes selon certains critères de recherche. On peut ainsi de manière automatique calculer la bathymétrie moyenne de chaque concession, voire même développer des modèles hydrologiques de calcul de flux de matières [1]. Une première fonction du SIG est donc naturellement de permettre l'édition d'informations stockées dans la base de don-

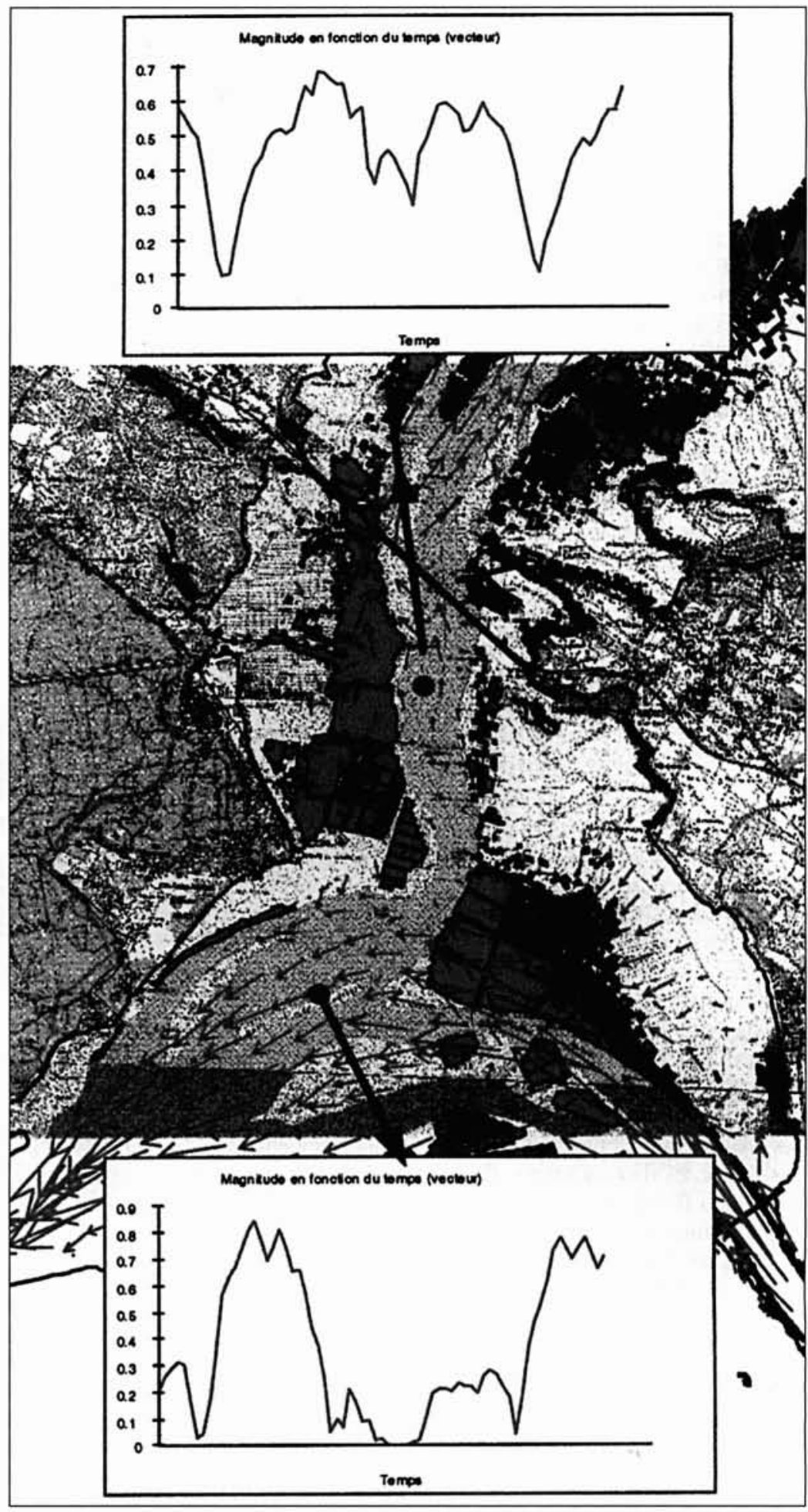

6. Carte du champ de courants de flot dans la partie sud du bassin de Marennes-Oléron et extraction des variations dans le temps du module de la vitesse en 2 points.

nées. Il sert également d'interface pour accéder, sélectionner et activer en mode sécurisé des procédures plus ou moins complexes d'analyse. Le principe de superposition des couches d'information existantes (thèmes) conduit à produire de nouvelles informations, comme par exemple l'estimation des durées d'immersion des zones d'élevage conchylicole situées dans un secteur géographique déterminé. Ce même outil d'aide à la décision a ainsi permis la détermination des 


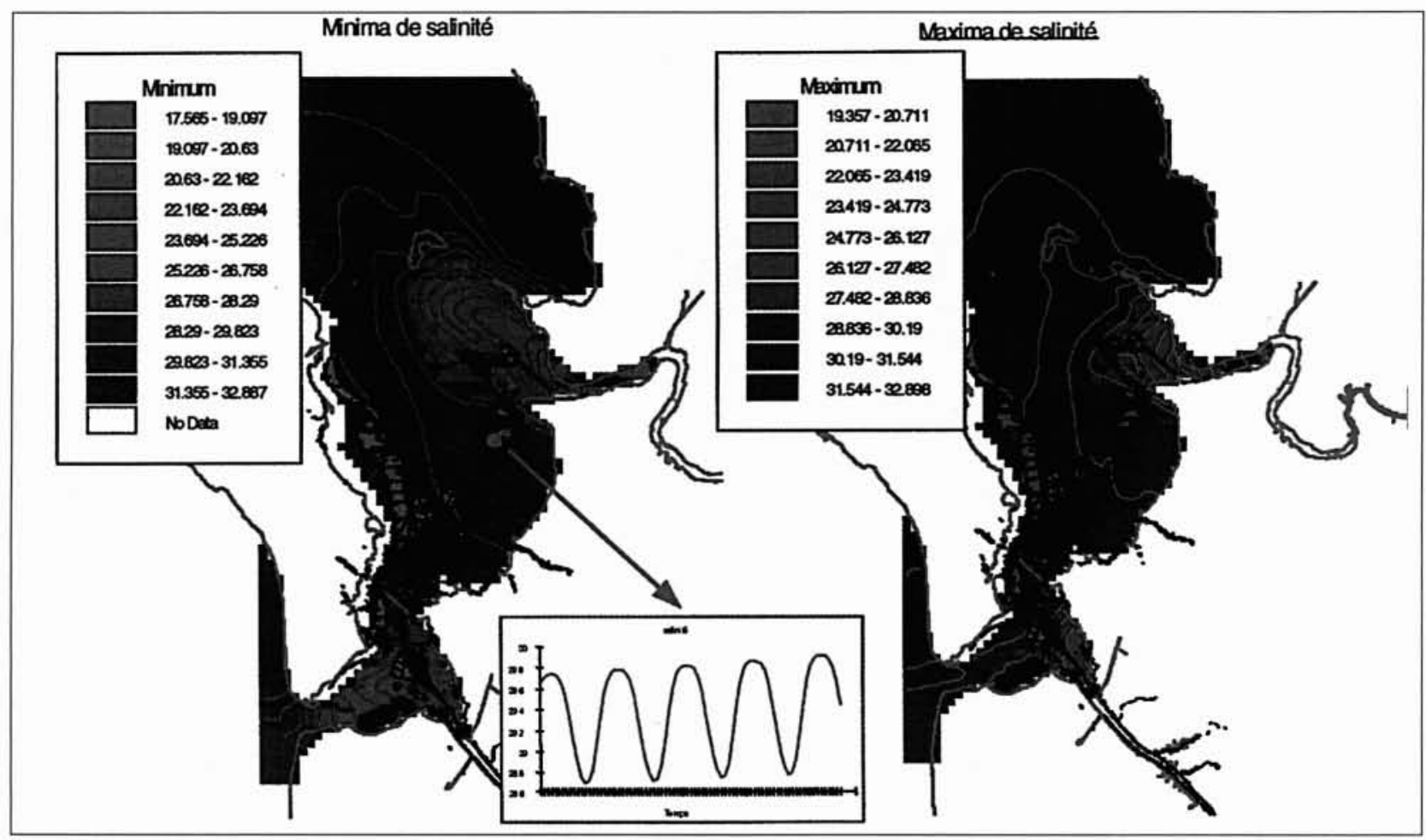

7. Cartes des minima et maxima de salinité pendant une simulation de vives-eaux avec des débits de rivière moyens et évolution dans le temps de la salinité en un point.

zones potentielles pour le développement de cultures d'huîtres en eaux profondes dans les Pertuis. Les bases de données provenant de réseaux d'observation sont de plus interfacées au SIG [12]. II est ainsi possible de superposer des observations répétées dans le temps (séries temporelles) d'un ensemble de descripteurs environnementaux aux thèmes géographiques véritablement spatialisés.

En ce qui concerne les simulations de l'hydrodynamisme et du transport, les résultats se présentent sous forme de fichiers contenant des champs de valeurs (salinité, hauteur d'eau) ou de vecteurs (vitesse du courant) pour des séries de dates (le pas de temps de sauvegarde des résultats étant de $10 \mathrm{mn}$ ) et pour l'ensemble des points du maillage de calcul (environ 13000 ). Les 6 scénarios présentés plus haut correspondent à autant de fichiers binaires au format d'accès direct, garantissant une recherche rapide des résultats. Une application a été mise au point pour réaliser un certain nombre de traitements définis dans une interface utilisateurs développée avec les outils de programmation du logiciel Arcview. Dans ce cadre, chaque champ définit soit un thème de type grille régulière de points pour les champs de valeurs, soit un thème de type point pour les champs de vecteurs. Ces fonctionnalités comprennent :

- La standardisation du référentiel géographique par conversion des coordonnées géographiques longitude, latitude du maillage initial en coordonnées Lambert.

- La définition d'une grille régulière sur la totalité ou sur une partie de l'emprise géographique du modèle, afin de cartographier les résultats de simulation.

— L'importation et la cartographie de la bathymétrie utilisée dans le modèle.

- La sélection, l'importation et la visualisation d'un fichier de résultats, d'un champ de valeurs/vecteurs et des dates de ce champ à importer. La salinité et la hauteur d'eau sont ainsi interpolées selon une grille régulière, la vitesse du courant représentée sous forme de vecteurs aux nœuds de calcul. Chaque date définit un nouveau thème ayant un ou deux attributs selon la nature du champ correspondant.

- L'exportation automatique des séries de cartes sous forme d'image au format GIF ou JPEG pour construire des animations.

- L'analyse des résultats sous trois formes :

— à partir des thèmes correspondant à des champs de valeurs ou de vecteurs :

- extraction des informations en un point donné,

- représentation sous forme de graphes et tables de séries temporelles,

- statistiques de base en ce point - moyenne, écart-type, minimum, maximum, pourcentage des valeurs comprises dans un intervalle,

- la cartographie des statistiques de base - moyenne, écart-type, minimum, maximum, pourcentage des valeurs comprises dans un intervalle, à partir des thèmes correspondant à des champs de valeurs ou de vecteurs,

- l'extraction des informations à une date donnée le long d'un transect et leur représentation sous forme de table et de graphe, à partir d'un thème correspondant à un champ de valeurs.

A titre d'exemple, les résultats d'une simulation des champs de courants, de la hauteur d'eau et de la salinité par coefficients de vives-eaux et en débit moyen de la Charente et de la Seudre sont analysés dans la zone du bassin de Marennes-Oléron. Le détail du champ de courant durant le flot est montré dans le sud du bassin et superposé aux secteurs conchylicoles (Figure 6). Les cartes des minima et des maxima de la salinité au cours de la simulation permettent de comparer globalement les échelles de variabilité spatiale et temporelle (Figure 7). L'application permet simultanément 


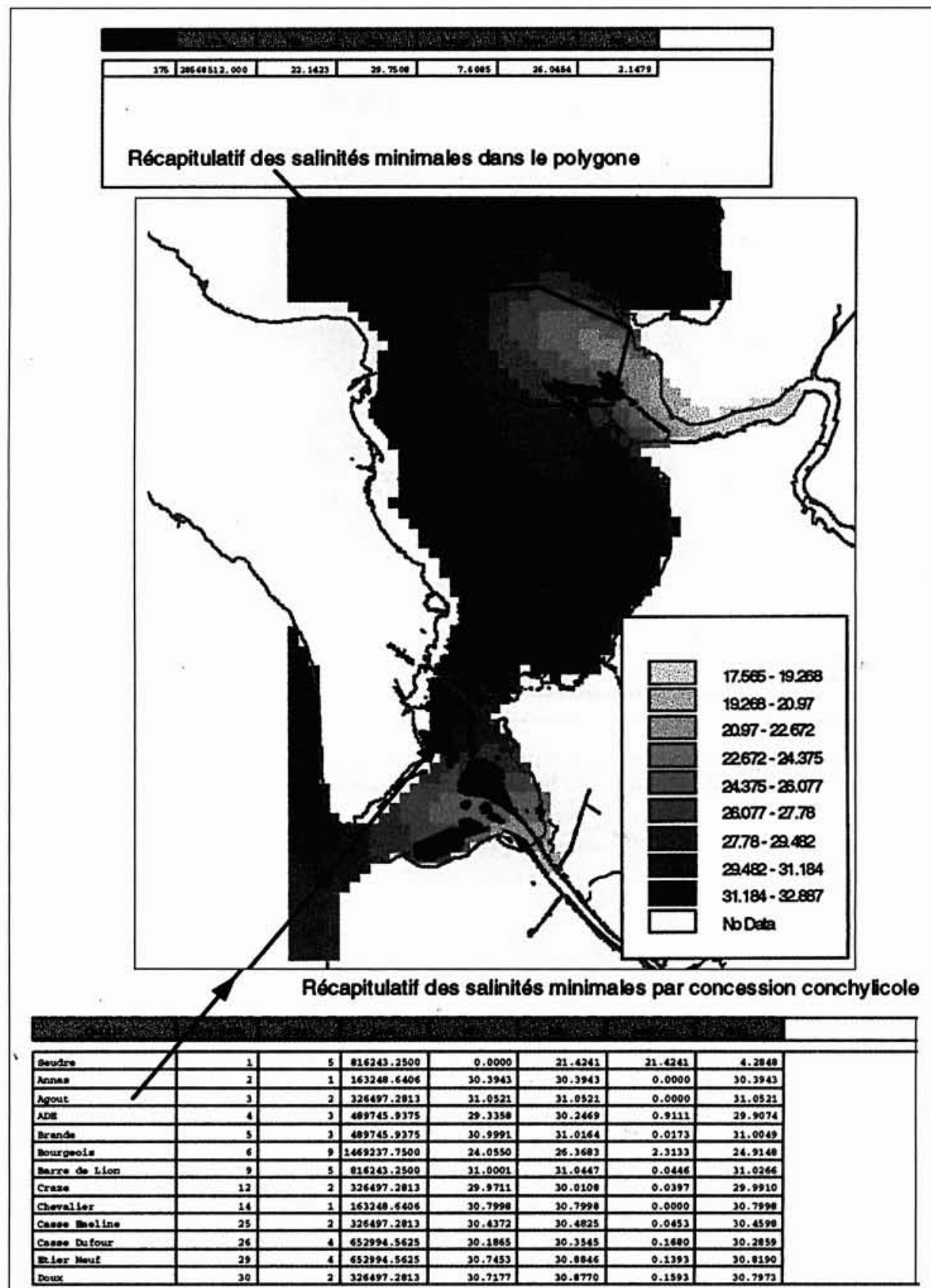

8. Récapitulatif des salinités minimales par secteur conchylicole - minima, maxima, moyenne, valeurs extrêmes - en particulier pour le banc Bourgeois au sud du bassin.

influence sur les panaches d'eau douce par exemple. De plus, un effort important a été consacré à la validation des résultats (pour plus de détail, [10]). Il en ressort une première conclusion importante concernant l'assimilation des données dont dépendent la mise en œuvre et la validation du modèle. L'hydrodynamisme est conditionné par la bathymétrie et des problèmes liés à l'imprécision, voire l'inexistence de ce type d'information se sont posés à différents niveaux :

- bathymétrie imprécise sur certaines zones du Plateau Continental auxquelles les conditions limites se sont révélées sensibles [10],

- bathymétrie ancienne et partielle pour l'embouchure de la Charente et inexistante pour l'embouchure de la Seudre, qui conditionne le volume oscillant dans des zones de mélange des masses d'eau d'origine continentale et océanique,

- bathymétrie partielle des zones de vasières, les hauts d'estran n'étant que peu renseignés.

Par delà l'étude de la variabilité environnementale par simulations, ce travail s'inscrit dans un contexte plus global de quantification, de visualisation et d'interrogation des paramètres hydrologiques susceptibles d'avoir une importance en matière d'aménagement et de gestion des activités du domaine maritime côtier. Les enquêtes auprès de plusieurs décideurs ont montré la sensibilité de certains secteurs en termes de qualité d'eau. Du fait de ses caractéristiques macroti-

d'afficher l'évolution de la salinité au cours du temps en des points sélectionnés par l'utilisateur. Enfin, il est possible d'utiliser les fonctionnalités de bases de données et de lancer des requêtes en croisant différents thèmes. Dans la Figure 8 , les salinités minimales au cours de la simulation sont superposées à la liste nominative des secteurs conchylicoles afin de récapituler les valeurs des minima de salinité par secteur.

\section{DONCLUSION ET PERSPECTIVES}

Les simulations ont permis de montrer la sensibilité du transport, de la hauteur d'eau et des caractéristiques de courant aux forçages dont les valeurs ont été définies d'après les statistiques disponibles - vent, régime hydrologique et coefficient de marée - et de hiérarchiser ces facteurs selon leur dales - fort marnage, surfaces de vasières importantes découvrant à marée basse, présence de marais protégés des surcotes par des digues, remise en suspension permanente des sédiments créant une forte turbidité, apports d'eau douce par la Charente et dans une moindre mesure par la Seudre température, salinité, vitesse de courant, transport de sédiment et d'éléments dissous et élévation du niveau moyen des eaux sont autant de facteurs élémentaires variables dans le temps et l'espace qui doivent être mesurés ou simulés. Audelà de la mise au point de modèles numériques, il s'agit de fournir une architecture et une organisation des connaissances disponibles sur le bassin de Marennes-Oléron, et cette étude contribue à divers efforts en cours. C'est ainsi que l'IFREMER a mis en place au niveau national des réseaux de surveillance de la qualité de l'eau et des coquillages 
depuis plusieurs années, qui permettent la détection en temps réel des zones et des périodes à risques sanitaires. Ces réseaux sont en cours de regroupements avec des réseaux complémentaires pris en charge par d'autres administrations (DDE, DDASS) dans un SIG côtier [12]. Cependant la diversité des connaissances disponibles, la multiplicité des sources et des formats de données imposent de constituer des ensembles de bases de données. Comprendre et décider ne seront effectifs que lorsqu'il sera possible de rechercher et recouper des informations correspondant à des événements particuliers concernant aussi bien les données mesurées que les informations provenant des modèles.

Il faut également insister sur la complexité du fonctionnement des écosystèmes marins et des interactions entre, par exemple, la conchyliculture et le milieu. Un exemple de modèle, validé et testé, a été présenté ici mais l'importance des processus biologiques pour les études d'impact a conduit à mettre au point divers modèles de production primaire, de capacité trophique, de remise en suspension des sédiments depuis une quinzaine d'années $[13,14,15,16,17,18]$. Le développement des bases de données accompagne ainsi un effort de compréhension et de modélisation des processus biologiques et physiques.

Ainsi, pour être opérationnelle, la modélisation doit s'appuyer sur un certain nombre d'actions nécessaires :

\section{Acquisition des données}

- La mise à jour de la bathymétrie des estrans et des estuaires est un prérequis à la qualité des prédictions,

- Les scénarios de simulation peuvent être définis sur des cas-types ou des conditions réelles. Dans tous les cas, les résultats dépendent de la définition des conditions aux limites (Golfe de Gascogne, estuaires) et des forçages (vent), - La quantification des débits et flux de matières réels en provenance du bassin-versant permet des simulations sur des cas concrets et non plus sur des cas-types.

- La validation des simulations repose sur les données disponibles - enregistrements en continu (salinité, hauteur d'eau, courant de marée) ou cartes synoptiques (panache de turbidité).

\section{Organisation des connaissances}

- La constitution de bases de données environnementales rend accessible la recherche et le croisement d'informations hétérogènes,

- Le développement des interfaces modèles/SIG et modèles/utilisateurs facilite le traitement de l'information et le travail de simulation.

\section{Prise en compte des besoins des utilisateurs finaux}

Les outils tels que modèles, bases de données, systèmes d'information géographique sont des éléments de connaissance scientifique qui doivent faciliter la fourniture d'avis en réponse aux questions des décideurs, aménageurs et acteurs locaux (zones favorables à la conchyliculture, impact des rejets aquacoles ou des rejets de dragage par exemple). L'intégration des résultats de simulation dans des outils d'aide à la décision complète ainsi les bases de données existantes.

\section{Remerciements}

Le présent travail s'inscrit dans le projet MAS3-CT97-0089 financé par la Commission Européenne (DGXII MAST Programme)

\section{Références bibliographiques}

[1] Bel. HASSEN M., 2000. Fonctionnement des marais maritimes atlantiques : échanges d'énergie et effets des flux aquacoles diffus. Thèse Univ. Bretagne Occidentale, 190 pp.

[2] LefebVRe S, 2000. Les cycles de l'azote et du phosphore dans un système aquacole intégré poisson-phytoplancton-bivalve : études expérimentales et modélisations. Thèse Univ. Nantes, $224 \mathrm{pp}$.

[3] Ravall-Legrand B., 1993. Incidences du débit de la Charente sur la capacité biotique du bassin ostréicole de Marennes-Oléron. Thèse Univ. Nantes, $171 \mathrm{pp}$

[4] Soletchnik, P., Faury, N., Razet, D., Goulletouer, P., 1998. Hydrobiology of the Marennes-Oleron Bay. Seasonal indices and analysis of trends from 1978 to 1995 . Hydrobiologia , 386, 1-3.

[5] Faury N., Razet D., Soletchnik P., Goulletguer P., Ratoskol. J., GARNIER J., 1999 : Hydrologie du bassin de Marennes-Oléron. Analyse de la base de données "Razlec" 1977-1995. Rapport IFREMER DRV/RA/LCPC/RST. 52 pages.

[6] SOGREAH, 1990. Estimation des débits d'étiage de la Charente à Saint-Savinien . Etude hydraulique.

[7] LHF 1990. Contribution au modèle global de l'écosystème d'un bassin versant et de la zone littorale associée ; Modélisation numérique des influences météorologiques sur l'hydrodynamique d'un bassin littoral peu profond. Programme Charente-Marennes-Oléron.

[8] Hervouet J.M., VAN Haren L., 1997. Recent advances in numerical methods for fluid flow. In Anderson M.G., Walling D.E. and Bates P.D. (editors) Floodplain processes, John Wiley, Chichesteer, 1997.

[9] Le Provost C., Genco M.L., Lyard F., Vincent P., Canalll. P., 1994 Spectroscopy of the world ocean tides from a finite element hydrodynamic model. J. of Geophysical Research, 99, C12, 777-797.

[10] Freissinet C., Sauvaget P., Bacher C, 2000. Environmental impaci assessment on the Marennes-Oléron Bay : operational mathematical modelling and GIS. International Conference ' Coastal Environment 2000', 18-20 septembre, Las Palmas de Gran Canaria, Espagne.

[11] Castaing P., 1981, Le transfert à l'océan des suspensions estuariennes cas de la Gironde. Thèse Univ. Bordeaux.

[12] Loubersac L., Salomon J.C., Breton M., Durand C., Gaudineau C. 1999. Perspectives offertes par la communication entre un modèle hydrodynamique et un SIG pour l'aide au diagnostic environnemental. CoastGIS'99, Brest, 9-11 Septembre 1999.

[13] Bacher C., Duarte P., Ferreira J.G., Héral M. et Raillard O., 1998 Assessment and comparison of the Marennes-Oléron Bay (France) and Carlingford Lough (Ireland) carrying capacity with ecosystem models. Aquatic Ecology, 31, 379-394.

[14] BACHER C., 1989. Capacité trophique du bassin de Marennes-Oléron couplage d'un modèle de transport particulaire et d'un modèle de croissance de l'huître Crassostrea Gigas. Aquat. Living Res., 2,199-214.

[15] Raillard O., Le Hir P., Lazure P., 1994. Transport de sédiments fins dans le bassin de Marennes-Oléron : mise en place d'un modèle mathématique. La Houille Blanche, 4, 63-71.

[16] Raillard O., Ménesguen A., 1994. An ecosystem box model for estimating the carrying capacity of a macrotidal shellfish system. Marine Ecology Progress Series, 115, 117-130.

[17] Guarini J.M., G.F. Blanchard, Ph. Gros, S.J. Harrison, 1997. Modelling the mud surface temperature on intertidal flats to investigate the spatio-temporal dynamics of the benthic micro-algal photosynthetic capacity. Mar. Ecol. Prog. Ser., $153: 25-36$.

[18] Guarini J.M., Ph. Gros, G.F. Blanchard, C. Bacher, 1999. La dynamique à court terme de la biomasse du microphytobenthos intertidal : formalisation mathématique. C. R. Acad. Sci. Paris. Life Sciences, Paris, Sciences de la vie, $322:$ 363-373. 